\title{
CRÉDITO Y BANCA EN EL MEDITERRANEO MEDIEVAL: LA QUIEBRA DEL CAMBISTA VALENCIANO FRANCESC DE PÁLS (1316-1319)
}

\author{
Juan Vicente García Marsilla \\ Universidad de Valencia
}

\begin{abstract}
SUMARIO
1. Los cambistas en la socieded valenciane en tomo a 1300.- 2. El negocio y el riesgo. Auge y caíde de Francese de Pals.
\end{abstract}

Corren los primeros dias de octubre del año 1316. Los ambientes mercantiles y financieros de la ciudad de Valencia se hallan especialmente revueltos. Se ha comenzado a propagar el rumor de que los negocios de la filial sevillana del cambista Francesc de Pals no generan más que pérdidas, debido a la insolvencia de su principal cliente, el noble rosellonés Jaspert de Castellnou, y aquellos que tienen cuentas abiertas en su taula se apresuran a reclamar sus haberes, temerosos de que una nueva quiebra bancaria les arrastre. Ello no hace sino precipitar los acontecimientos, y el dia $9 \mathrm{Pals}$, incapaz de satisfacer a sus numerosos acreedores, huye y se refugia en el cercano monasterio de Sant Vicent de la Roqueta. Hasta alls le persigue una muchedumbre enfurecida, dispuesta incluso al linchamiento, que montará guardia día y noche en torno al convento exigiendo que le sea entregado el presunto estafador. Sólo al día siguiente, más serenos los ánimos, la mediación de los fiadores del cambista arruinado -los influyentes banqueros Jaume Magraner y Bernat Planell- permitirá llegar a un acuerdo ante notario. Por el cuarenta y siete personas se habrán de repartir los bienes de Francesc de Pals, asI como los ingresos que fueran generando los créditos que éste habra a su vez facilitado, y las empresas comerciales en que se hallaba

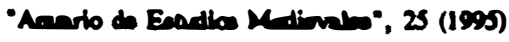


inmerso, a cambio incluso de renunciar a cobrar una parte del capital invertido, con tal de no perderlo todo. Más de tres años tardarfa en liquidarse totalmente la deuda, quedando registrada la dificil gestion de los síndicos encargados del caso en un volumen especial de la serie del Justicia que contiene datos realmente preciosos para un mejor conocimiento del mundo de la banca en la Valencia de principios del siglo XIV'.

\section{LOS CAMBISTAS EN LA SOCIEDAD VALENCIANA EN TORNO A 1300}

La quiebra de Francesc de Pals no fue, sin embargo, un hecho aislado, ya que tanto Valencia como Barcelona e incluso los grandes centros financieros de Italia atravesaban por sus primeras dificultades de cierta consideración, que se manifestaban en un auténtico rosario de bancarrotas por toda la ribera norte del Mediterráneo ${ }^{2}$. Esta coyuntura critica, cuyas manifestaciones y causas estamos aún lejos de comprender en su totalidad, es especialmente desconocida en Valencia, donde los estudios sobre la banca privada medieval son casi inexistentes ${ }^{3}$. Por ello creemos necesario un

\footnotetext{
'Se coneerve en el Archivo del Reino de Valencia (en edelente ARV Justcia de Vallencia 22, con el titulo de Pleits civils d'en Francesc de Pals. Cambiador de Valincia. El hocho de que eate proceso $x$ eneventre en la cerie del Justicia 20 debe a que eate oficinl municipal fue

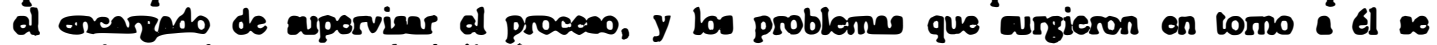
cometionon siempre a w juriadicción.

${ }^{2} Y$ e en 1256 e habia producido la primen crisis bancerin en Genove (vid. R. S. LOPER, La prima crisi delle barca de Genove (12501259), Milan, 1956). En 1298 quiebra la bance Bonaignori de Sien (R. do ROOVER, The rise and fall of the Mediai Bark, 1397-1494, Cambridge Mees., 1963, pp. 2-3). Y en 1310 to hacen los Ricciandi de Lucca, ante he insolvencie de we mayores clientes, los reyes de Ingleterre (M. PRESTWICH, lealian merchares in the lase thirteenth and carty fourteensh concury Engloand, en The Dawn of the modem barting, New Haven-Londres, 1979, pp. 77-104, p. 78; y tumbien, R.W. KuEUPER, Barkers to the Crown: The Ricciandi of Lucca and Edward I, Princeton, 1973). Las dificultedes por las que atraveanon los cambiren calnhnes en 1298-1299 en S. P. BENSCH, La primera crisis bancaria de Barcelona, "Anurio de Estudios Medievales" 19, (1989), pp. 311-327. Las primeras fallides de la bance valencians dathn al menos de 1299, como mis edelante veremos.

'Se limitan a una breve note a partir de una fuente normative corno ea el Aureum Opus de La ciuded, a cargo do A. GNRCY SANZ, La Bance en los siglos XVII y XVV seguin el Aureum Opus, "Bolotin de la Socieded Cenellonenes de Culturn" XXXII (1957), pp. 201-205; y a gunos detos en el arésulo global de M. RrU, La berca i la socielas a la Corone d'Aragb, a finals de l'Edar Mifjora i comongann's de la Modema, "Acta Medinevalin" 11-12 (1990-1991), Pp. 187-244; y en le obre clínica de A. P. UsHER, The early history of deposit barling in Medisernerear Eunope, Nuova York, 1943. Man atención ha recibido en cambio la caula de convis municipal, con estudio como los de M. PERIS y FUENTEs, Orfgens de la Taula de
} 
primer acercamiento global al mundo de los cambistas, el crédito y la finanzas en el ámbito valenciano desde la fundación del reino hasta estos años iniciales del Trescientos, que sitúe los acontecimientos en su contexto preciso.

Asl, la verdadera acta fundacional de la banca valenciana dentro del mundo cristiano occidental data del año 1239 , y es la creación, por Jaime I, de una zona reservada para las tablas de cambio y los obradores textiles en el solar de la ciudad conocido como Vall del Paradis. Este lugar, delimitado por la zapatería, la carnicerfa y el lienzo de muralla junto al cual se dispondria cada jueves el mercado, acababa de configurar el verdadero pulmón económico de la urbe, situado en su parte sur, en la parroquia de Santa Caterina. El rey garantizaba además, a cambio de un módico censo de dos morabatinos al año por obrador, la exclusividad de los vecinos de este nuevo Carrer dels Canvis en los intercambios monetarios". La posterior acuñación de moneda privativa del reino, el real de Valencia, en 1247, confirio incluso mayor importancia al papel de los cambistas de la ciudad, que eran a la postre los que habrian de fijar la paridad con el resto de divisas que circulaban por sus mesass. Pero, siguiendo las prácticas que desarrolla-

Vallencia, "III Congreso de Historia de la Corona de Aragón", (Valencia 1923), I, pp. 503-518; S. CARreres Zacares, La primitiva Taula de Cambis de Valzncia, "Boletín de Sociedad Cartellonense de Culture" 25 (1949), Pp. 708-768, y A.E. SAYOUS, La Techrique des affaires, sa génese. II: Une caisse de dêpots: la "Table des Changes de Valence (1407-1418), "Annales" 6 (1935), pp. 133-137.

'Libre del Repartiment de Valzencia, edición de Antoni FERruNDO, (Valencia, 1979), entrada 1217 del año 1239. La doneción ee hace a un grupo formado por 34 pernonas. J. RODRIGo PERTEGAs localizabe esta zona entre las actuales calles del Trench y de las Mantas en La urbe valenciara en el siglo XVV, "III Congreso de Hirtoria de la Corona de Aragón", cis., pp. 279-374, pp. 331-332. No obstarle, el rey no debió reopelar duranie mucho tiempo el monopolio del cambio que oterntaba eata zona, ya que en 1263 so habla ya de las "rabulace cambii novis et veteris" (Archivo de la Corona de Aregón (en adelante ACA), Reial Cancelleria, Reg. 14, fol. 12 v.). Dentro del mimo Repartiment aparocen, por otre parte, algunos establecimientos individuales de cases, huertos o molinos a cambietns en Valencia, Rusaafa, Atzira y Sagurs, corno las que rociben los judios Abrahim y Açat, y los cristianos Guerau Eacrivd, Domingo y Bemat (entrades 616, 2.092, 2.131, 2.132, 2.349, 2.351, 2.380, $2.485,2.953,3.219,3.249$ y 3.724$)$.

'El centuto de creación del real valenciano es del 8 mayo de 1247 (8 idus madii), y eparcece rocogido en el Aureum Opus Regalium Privilegionum Civitaris et Regni Valentie, Jacobi primi, cap. XXII, p. 77 de la edición de L. AlANYA, faceímil de la de Gumiel de 1515 (Valencia, 1972). En ella el rey eatablece una tabla de su propiedad durante cuarenta días en la que so extipulan los tipos de cambios iniciales, que luego deberian quedar a criterio de los cambistas ("..secundum que denarii realium cum predictis monetis in tabulis campsorum ad cambium valuerint"). Acerca de la moneda en peta primere Epoce vid. F. MATEU y LLOPIS, Sobre la poltrica monetaria de Jaime I y las aculleciones valencianas de 1247-1271, "Anales del Centro de Culture Valencinsa", 1947, pp. 233-261. 
ban ya la mayoría de ellos en sus hugares de origen -puesto que todos eran, de alguna manera, inmigrantes- estos profesionales del cambio no se limitaban a dicha función, sino que acepeaban ya dinero en depósito de terceras personas, con el cual expeculaban e invertion en los más variados negocios. De esta manera, se puede aplicat propiamente el término de banqueros a la práctica totalidad de las cuarenta y meve personas que hemos detectado hasta ahora en las fuentes como compsores Valencie entre el momento de la conquista y $1320^{\circ}$.

Precisamente es de la actividad cambiaria manual de la que actualmente poseemos menos datos, al ser una operación realizada de forma inmediata sobre las mesas y que no suele registrarse por escrito. Aún asi, algunas referencias indirectas permiten afirmar que en los establecimientos bancarios valencianos se deblan manejar importantes sumas de numerario, como lo demuestran los cuatro mil sueldos en metálico que Marti Serra denuncio en 1273 que le hablan sido robados de su boneta cuando volvía de la taula de canvi'. La especulación con el valor de las distintas monedas, y el cobro de pequeñas comisiones, constitulan la escasa ganancia de los cambistas en este tipo de operaciones, que eran, con diferencia, las menos

\footnotetext{
La fuentes utilizadas son muy varindes, e incluyen los escasoo protocolos notariales de ha tpoce, conservados en el ARV; la serie del Justfcia del mismo anchivo; la Reial Cancelleria y el Reial Parrimoni del ACA; loo Mormals de Consells del Archivo Municipal de Valencia (en edelante AMV), y los pergaminos de la catedral de Valencia (en edelante ACV). El listado, por riguroes orden cronológico de aperición en las fuentes es el siguiente: Abrahim, jueu; Guerau Eecriva; Domingo; Açat, jweu; Bemat; Domingo de Agde; Berenguer Dalmau; Guillem de Perelada; Mart' Serra; Romeu de Soler; Guerau Martí; Guillem Amau; Guillem de Senta Cilia; Ponç Fibla; Jaume de Sentboi; Bemat Ferrer padre; Berenguer de Guarea; Pere Guillem Catald; Jeume Magraner; Ramon Soler, Berenguer Gudrdia; Berenguer de Tovia; Bartomeu Arquell; Franceac Alberola, Andreu d'Acrimonle; Pere Muntanyola; Bemat Planell; Ferrer Oller; Jaume de Soler; Ponç de Soler, Pere de Pratboi; Bemal de Canet; Bernat Derani; Bernat Ferrer hijo; Bemat de Llemena; Bartomeu Ceriol; Francese Solanea; Amau Guillem Cataln; Benat de Vilalba; Francese Planell; Francesc de Pala; Franceac des Man; Mateu de Boneacombea; Pere Miracle; Bernat Berga; Ramon de Poblet; Pere Vieder, Guillem Mir y Guillem de Sarrì.

'ACA, Reial Cancelleria reg. 19, fol. 97. Xiva 18 de diciembre de 1273. Transerita en A. HUTCI y M.D. CABUNES, Colección diplomérica de Jaime I el Conquislador, Valencin 19161922, tomo III, pp. 424425. Serse denunciabe edeml la deanparición de "..eltres comee valente mil colidos" y de que "..tolgeren-me un coltoll e mefruren-me dues mafres morthlo e feriren-me de moltz colpe de pilotes de plom e de pedres..". Peac a la probable exngeración de exte

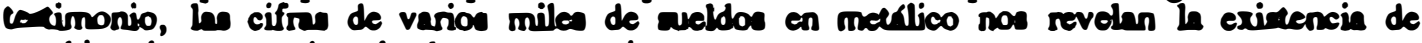
cambios de envergedure hechos en monode concares.
} 
remuneradoras de cuantas realizaban". El resto de negocios implicaba en cambio un mayor movimiento de capitales, los cuales deblan quedar durante cierto tiempo a recaudo del banquero. Por ello era obligatorio que éste llevara un libro de cuentas o capbreu, que a veces podia desdoblarse en dos: uno de transacciones diarias, y otro, el major, donde las operaciones apareclan agrupadas por cuentas personales?. Por desgracia no se ha conservado ninguno de estos registros en Valencia, y sólo podemos afirmar su existencia a partir de alusiones que a ellos se hacen en otras fuentes. AsI el libro de la taula de Francesc de Pals aparece perfectamente descrito en el proceso, y, por lo que parece, debra ser un simple registro de deu i deig en el que los contratos eran anotados por un empleado del cambista uno debajo de otro por simple orden cronológico ${ }^{10}$.

\footnotetext{
'En Barcelona a finales del Trescientos las ganancias de los cambistas en eatas operaciones rondaben el 1'78\%, ecgún R. CONDE, Las acrividades y operaciones bancarias de la banca barcelonesa trecentista de Pere Descaus y Andreu d'Olivella, "Revista Española de financiación y contabilided" XVII (1988), pp. 115-187, p. 146. En Valencia, por ejemplo, el cambio extablecido para el toumois por orden real, tanto en 1247 como en 1309 (AMV, Manuals de Consells A-1, fol. 29) era de 15 dineros de Valencia, mientras que el cambista Bernat Planell 2 los cobraba al baile a 15'5 o 16, es decir, que obtenia unos beneficios de entre el 3'33 y el 6'66\% del capital (E. GONZALEZ HURTEBISE, Libros de Tesorería de la Casa Real de Aragón, tomo I, Barcelona, 1911, pp. 348-349 y 367-368).

'El catudio de los sistemas de contabilidad medieval a partir de este tipo de libros ha partido cobre todo del caso italiano. Vid. por ejemplo R. de ROOVER, The Development of Accounsing prior to Lucca Paccioli according to the Accouns Books of Medieval Merchanis, "Business, Banking and Economic Thought", Chicago y Londres, 1974, pp. 119-128; y CH. M. de la RONCIERE, Un changeur florensin du trecenso: Lippo di Fede del Sega (1285 env.-1363 env.) Parí, 1973, especialmente sobre la contabilidad la páginas 11-21.

${ }^{10}$ En el fol. 78 v. del volumen del proceso se refiere cómo los albaceas de Romeu Dalmau presentaron como pruebe de las 250 libras que se les adeudaban: "..I libre de la taula de cambi del dit en Francesch de Pals, en paper ecrit, ab cubertes de cuyr vermeyl emprentades deswis amb empremies morieques, lo qual libre to appellat libre cinque del ayn de MCCCXV, en la eegona carta del qual libre era escrita la infraegüent scriptura, en lo comensament de la dita segona carn:

En nom de Déu sia, e de la Verge Madona Sancta Maria e de Tots los Sante de Pareyí, yo, Bernat Gri, contare a ecriure equeat present libre a obs de la taula de Francesch de Pals, e fo a VIII dies de juliol en l'ayn de nostre senyor MCCCXV.

(Libre V d'en Bernat G.)

Del qual libre apres de CLXXXV cartes d'aquell libre fon prese e treyta la infrasegüent eacriptura:

Deig a n Pere Guerau, rector de Sent Nicholau, e a frare

Bermal Saporta e a'n Jacme de Riusech, mamesor d'en

Romeu Dalmau que hic muda de compte del dit en Romeu en

CCCIII c'.... .CCL $1 . "$

Es probable que la mayoria de los cambistas contaran con ayudantes o escribanos que llevaban las cuentas, aunque a voces eran apuntadas por ellos mismos, como hizo Bermat de Llemena en 1295, al recibir 50 libras de Jaume Perpinya "..quas ecripai vobis manu mea propria in libro moe campeorie.." (ARV, Protocolos Jaume Mart 2.631, 12 de diciembre de 1295).
} 
Lo primero que se debra reflejar en estos libros eran las cantidades que el cambista recibra de sus clientes, llamadas comandes de dipdsit o simplemente comandes, que en ocasiones se podian formalizar también ante notario, por lo que algunas nos son conocidas". En ellas no se consigna más que el reconocimiento del banquero de haber recibido en su taula una cierta cantidad de dinero que deberá reintegrar en cuanto le sea requerido por el titular de la cuenta. Las cantidades que se reflejan en los protocolos son realmente importantes, casi siempre superiores a los 2.000 sueldos y a veces hasta de 10.000 , posiblemente porque para los pequeños ingresos no serla necesario acudir al notario ${ }^{12}$. Los especialistas discuten aún sobre la finalidad de estas comandas: ¿el depositante buscaba sólo seguridad para sus ahorros o recibra ya en esta época algún tipo de interes?. En buena parte, la respuesta a esta pregunta depende del margen de maniobra de que dispusiera el banquero para negociar con las sumas que aceptaba. De hecho existen algunos contratos en que la cantidad entregada aparece encerrada en bolsas lacradas que se han de devolver intactas, por lo que la función del cambista se reduce aqur a la custodia del dinero, eliminando toda posibilidad de lucro por ninguna de las partes ${ }^{13}$. Pero esta cláusula sólo aparece raramente, al menos en cuanto a las comandas aceptadas por cambistas se refiere, y lo más normal era que estos tuvieran potestad para emplear las sumas que se les habran confiado en negocios propios. De ahl la importancia de algunos privilegios otorgados por el rey prohibiendo que se embargaran cuentas de las taules por deudas de los depositantes, como el que recibió Romeu de Soler en 1275 , lo cual daba mayor seguridad al banquero a la hora de invertir el capital ajeno de que disponfa ${ }^{14}$. Sus clientes en estos casos

\footnotetext{
"En los protocolos de Valencia del ARV de 1285 a 1308 -diez en lotal- hernos localizado once comandas realizadas en las mesas de nueve cambistes diferentes.

${ }^{12} 10.000$ sueldos as la cantidad que el noble Jaume de Romans ingreas en la mean de Pere de Muntanyola el 21 de diciembre de 1296, y probablemente no lo hizo en metalico, sino modiante una dita o transferencia deade los bancos de Bartorneu Arquell y Bemat de Llemena, en las cuales Romanl canceló was cuentas, procienmente por valor de 10.000 s., un día despué (ARV, Protocolos Desconocido 11.178).

${ }^{13}$ Ea el caso de la comanda que el 26 de mayo de 1335 realizo el obispo de Valencia, Ramon Gastó, en la laula de Bernat Joan, de 10.094 s. 6 d., "..in tribus sacciis carrapis sigillatis.." (ARV, Protocolos Aman de Casesvelles 2.820). Según A.P. UsHER, op. cit. p. 9, esto es lo que el Derecho Romano reconoce como "deposito gracioso". R.C. MUEURR, en The Procurasori di San Marco and the venetian credit market, Nueva York, 1977, p. 168, los observa en Venecia y los denomine regular deposis.

${ }^{14}$ ACA, Reial Cancelleria, reg. 20, fol. 314 v. (kalondas fobruarii 1275).
} 
recibirfan una parte de los beneficios conseguidos o un interés fijo previamente pactado, aunque los dividendos obtenidos por los impositores nunca aparecen en los contratos, probablemente ante la posibilidad de que dichas ganancias fueran consideradas como usura ilicita ${ }^{15}$.

Los titulares de las cuentas gozaban además de la comodidad de poder realizar sus pagos por medio de dites o transferencias a otros clientes del mismo banco o incluso de otros, con lo que evitaban las molestias y peligros que suponia acarrear grandes cantidades de monedas. Las mesas de los cambistas se revelaban de esta manera como instrumentos perfectos para ciertas administraciones, como las nobiliarias o eclesiásticas, 0 incluso para el reparto de una herencia o la gestión de la tutela de un menor ${ }^{16}$.

No obstante, el gran beneficiario de la acumulación de capital era, por supuesto, el propio cambista, ante el que se abria un abanico bastante amplio de inversiones altamente rentables. La primera alternativa podía ser el mercado local del crédito, en el que los banqueros jugaron un papel destacado. Hacia el año 1300 el tipo más habitual de préstamo era el que aparece en la documentación con el simple calificativo de mutuum. En él, bajo la formula confiteor me debere vobis ratione mutui, el deudor reconocla haber recibido una suma cuyo plazo de devolución podía variar entre una

\footnotetext{
${ }^{15}$ Como ocurrió en Venecia en 1340, cuando los clientes del cambista Donato Quintavalle fueron scusados de usura. En eath ciudad hacia los años de 1330 se han detectado intereses entre el 5 y el 7\% anual (R.C. MUEUER, op. cit., Pp. 175-177). En las comandes valencianas ctudiadas precianmente en el único caso en que se habla expreannente de lucro vel usura -annque ain especificar cuánlo--, es en una comande que hace un cambista, Berenguer de Guares, - ols pereona, Amau de Senent, de 4.600 s. a devolver en un año (ARV, Protocolos Desconocido, 11.178, 22 de diciembre de 1296). Este caso, como la mayoría de las 72 cormanda entre particulares que hernos podido constaters hasta 1305, encubre en realidad un pretanno unuraio.

10Aar por ejemplo en 1308 Gombeu Eiximen, señor de Arenós, y Eiximén Péreg de Montomto, como mamessors de Sarre Femendig Dieg rociben del notario Nicolau Puig los bienes de la difunta en la kaula del cambira Bernat Derani, donde permanecerán mientras los dminirsen (ARV, Prosocolos Jaume Mart, 2.871, 23 noviembre (VIII kalendas decembris)). El miemo rey Alfoneo el Benigno, y Baromeu de Pont, como tutores de la esposa de Guillem de Sont Melío, contralizaban la gextión de au herencia desde la mesa de Guillem de Senta Cilia (ACA, Reial Cancelleria, reg. 74, fol. 20, 17 de noviembre de 1287 (15 kalendas decembris). También los pagon que debía realizar Gil Ruiz de Lihori, señor de Sot de Xera, los hacía en la banca de Bernat Planell, como la entrega de au parte del diezmo al obispo de Valencia, convittente en 200 s. en 1296 (ARV, Protocolos Desconocido 11.178, 9 de febrero (5 idus forrenii).
} 
semana y cuatro años, pero que habitualmente no superaba los seis meses ${ }^{17}$. Se trataba por tanto de créditos a corto plazo, ideales para unos cambistas de solvencia limitada, que deblan estar pendientes de las posibles peticiones de reintegro de sus clientes. En cuanto al interés cobrado en estos préstamos, que nunca aparece de forma explicita, deberfa ser como minimo del 20\% anual autorizado, y frecuentemente superior, lo que podía llevar a acusaciones de usura ante los tribunales civiles o eclesiásticos ${ }^{13}$. También en ocasiones los prestamistas exiglan una prenda con la que se pudieran cobrar en caso de impago, que podía consistir en una mula o un rocín para los deudores más humildes, o en preciadas joyas en los créditos concedidos a la nobleza o a los monarcas ${ }^{19}$.

En total, de los 94 mutua registrados en los protocolos de esta época, solo en dieciocho el acreedor era un cambista -el 19'14\%-; pero en cambio de los $59.009 \mathrm{~s} .2 \mathrm{~d}$. de capital total a que estos préstamos ascendieron, 36.677 s. $8 \mathrm{~d}$. fueron suministrados por estos banqueros -el 62'15\% -, lo que demuestra su predominio especialmente en las operaciones de mayor envergadura. AsI el mayor prestamista del momento parece ser Ponç Fibla, protagonista de seis contratos que suman un capital de $15.257 \mathrm{~s}$. $8 \mathrm{~d}$., de los

\footnotetext{
17Sobre una mueatre lotal de 86 musua extraidos de los protocolos de 1285 a 1308, w6lo 34 expocifican el plazo de devolución, y con los aiguiencea:

cernana 14 meses 24 años 4

2 eernane 25 meses 1

3 cernanas 36 meses 3

1 mes 47 meses 1

2 meses 511 meses 1

3 menes 41 año 4

Eate debin eer el tipo predominante de prestamo al consumo en toda la Europa meditertinea en eath Epoca, como ocurre por ejemplo en Montpellier (vid. K.L. REYERSON, Business, Banting and Finance in Medieval Monspellier, Toronto, 1985).

${ }^{12}$ Ferrer Oller, saenrrere cambiador, y su mujer Francesca fueron acusados de haber "hails logre" y no declararlo, por cinco vecinos anle los jueces diputados por el rey, Pere Gomir y Ramon de Sentloyr, el 20 de mayo de 1299, y hubieron de pagar mil morabatines de oro de multa (ARV, Prolocolos Jaume Mart 2.811, 12 kalendas junii). La tasa de interts permitida en los Furs en 1241, y de nuevo en 1283, era de 4 dineros por libra (20\%) pare los judíos y musulmanes, siendo ampliado pare los cristianos en el texto latino (Vid. Furs de Valencia, ed. de G. Colon y A. GARCLA, Barcelona, 1983 núbrica XIV del libro IV). Recogido también en J. CORTES, Liber privilegionum civieatis et regni Valensie. Edició crtica $i$ andlisi cadicoldgica. Teais Doctoral inédita, Univernitat de Valéncia, 4 vols., 1987. El "De statutis usurarum" en vol. II, pp. 25-31.

"Con aperas dos días de diferencia se puede regintrar un prealamo que el canvista Bemat Ferrer hizo al fuster Gil Eiximén d'Aynar de 400 s. con un rocín de pelo rojo ensillado y herredo como penyora; y el crédito que Bemat de Llèmena hizo al noble Amor Dionisi, señor de Canals, de 1.200 s. a seis meses, dejando en prenda 8 anillos de oro, 5 de ellos con zafiros y 3 con rubies (ARV, Protocolos Desconocido 11.178, 15 y 17 de diciembre de 1296).
} 
que destaca el crédito de $10.000 \mathrm{~s}$. que habra realizado a la aljama hebrea de Valencia ${ }^{20}$. El resto de su clientela se recluta especialmente entre las clases mercantiles, aunque incluye también un préstamo al almirante Roger de Llúria, que éste carga sobre su señoría de Castellnou. Más especializado en los créditos a la nobleza parece Bernat de Llemena, de cuyos 10.650 sueldos de capital prestado en siete operaciones, más de la mitad corresponden a dos contratos que tienen como prestatarios al señor de Canals y a Berenguer de Vilaragut, titular de Sollana y Albaida ${ }^{21}$.

No obstante, las inversiones de estos cambistas desbordaban con frecuencia el marco de la ciudad y su hinterland, implicándose a menudo en operaciones mercantiles a escala internacional. Algunos incluso poseran sus propias naves con las que podran realizar expediciones por el Mediterráneo; aunque la mayorra participaban en estas empresas mediante comandas comerciales en las que el cambista era el socio stans, el cual proporcionaba el capital a un mercader que realizaba el viaje, quedándose el banquero con $2 / 3$ de los beneficios ${ }^{2}$. El comercio de paños, ya fueran lombardos y occitanos, era quizás el negocio más lucrativo, y por ello algunos cambistas intervinieron en él de forma activa. Bernat de Llemena, por ejemplo, protestaba en 1295 porque dos balas de draps de França que importaba por via terrestre hablan sido embargadas por el lleuder de Tamarit de Litera; y

\footnotetext{
${ }^{20}$ En 1299 ya había hocho cesión de eate credito a Pere Amalric, de Morvedre, y ante él se presentaban los adelantados de la aljama (ARV, Protocolos Jaume Mart 2.811. (6 nonas madii).

215.400 \&. prestó a este último el 18 de febrero de 1297, y también salieron de su taula otros 3.032 s. 8 d. que Vilaragut rocibió del miles Gil Marín d'Entença el 31 de enero anterior. La deuda de Amor Dionisi, señor de Carals, ascendía a 1.200 s. (datos de ARV, Protocolos Desconocido 11.178). La liquidación de la deuda de 2.000 s. de Roger de Llúria a Ponç Fibla la realizó el mismo alamí de Castellnou el 13 de febrero de este mismo año (misma referencia anchivistica). Los demás créditos fueron facilitados por Jaume Sentboi (una operación, 100 s.); Bernat Ferrer (dos, 2.070 s.); Bernat Planell (una,4.000 s.); y Berenguer de Guares (una, 4.600 8.).

${ }^{2}$ Ejemplo de cambista con nave propia es Jaume Sentboy, que poseía una "barcham meam armatam cum XVII rimorum cum tota sua exarcia et apparatibus quam habeo in rambula Valencie", la cual sirvió como aval de un crédito a su socio el argenser Pere Terrer (ARV, Prolocolos Jaume Mart, 2.631, 22 de febrero de 1296). Ponç Fibla en cambio confiaba a los mercederes mallorquineo Amau de Caseo y Joan Pont 500 s. parz un viaje que realizarían a Honein, en el norte de Africa, quedándose Fibla con 2/3 de los beneficios (ARV, Protocolos Desconocido 11.178, 13 de diciembre de 1296). Los cambistas podian incluso participar de esta manera en campañas de coreo, como hizo Garcia Eiximenis en 1357, patrocinando la actividad de Domingo Roures, que fue ajuaticiado ese año por haber roto una tregua con el rey de Granada, por lo que el cambista hubo de pagar 3.000 s. de composición (ACA, Mestre Racional 1.074, fol. 24).
} 
un año más tarde lo encontramos comprando por 60 libras a Berenguer Maç $\sigma$ tres paños tintados y tres blancos de Narbona ${ }^{23}$. Otros prefieren comprar la mercancla a drapers languedocianos que tienen abiertos obradores en Valencia, muchas veces para revenderlos por las villas del reino, convirtiéndose Valencia en un importante centro redistribuidor de los tejidos france$\operatorname{ses}^{24}$.

Las fluidas relaciones con los mercaderes de más allá de los Pirineos facilitarán a su vez la articulación de un mercado de capitales a larga distancia, en la que los banqueros valencianos

servian como corresponsales en los contratos de cambio negociados con plazas de Francia o Italia ${ }^{25}$.

Sin embargo, los negocios que mayor movimiento de capital implicaban para los cambistas eran aquellos que tenian que ver con la Corona. En efecto, desde muy pronto los vínculos entre la monarquía y los banqueros fueron muy estrechos, dadas las crecientes necesidades de numerario de unos reyes que pretendlan centralizar en su persona el poder político, y extender sus dominios por el Mediterráneo. De esta manera, en una etapa en que la percepción de impuestos cơmienza a ser cada vez más regular, los cambistas paliarán la lentitud del proceso recaudatorio adelantando sumas al rey, que luego los súbditos debran ingresar en sus taules. Asf, los 30.000 s. que los judios del reino deblan pagar como subsidio a una

\footnotetext{
${ }^{23}$ La protesta ante el lleuder en ACA, Reial Cancelleria reg. 89, fol. 56 v., 12 de enero de 1295. La compra a Maçó en ARV Protocolos Desconocido 11.178, 14 kalendas januarii 1296 (19 diciembre).

${ }^{24}$ Asi Pere Guillem Catall daba en comanda a Jaume Miquel doce draps d'or en 1292, para que los vendiere en Gandía (ACA Reial Cancelleria reg. 86, fol.s 114 v.-115 r., 13 de mayo de 1292). Bemat Degani, por su parte, vendia al draper Guillem d'Esplugues en 1297 cinco paños tintados y uno blanco de Nábona por 1.147 s. (ARV, Protocolos Desconocido 11.178, 6 de febrero). Mientras Francesc de Solanes compraba al narbonés Andreu de Termens paños por valor de 91 s. en el obrador "quod tenebal in draperie Valencie" (ARV, Protocolos Jaume Mart 2.871, 19 octubre 1308). Sobre la importación de tejidos del Midi en esta Epoca vid. G. ROMESTAN, Les marchands languedociens dans le royoume de Valence pendans la première moitie du XIVZ siecle, "Bulletin philologique et historique", (Paris, 1969), pp. 115-192 (hay traducción catalana en A. FURIO (ed.), Valencia, un mercal medieval, Valencia, 1985, pp. 175. 263).

${ }^{25}$ Un ejemplo son los 6.000 s. que recibe Bemat Ferrer de Bemat de Sentacilia, draper, por mercancias que éste adquirió del italiano Giacomo Maurizio. O, a la inversa, los 2.320 s. que Ponc Fibla entrega a Amau Escriva, miles, pare pagar al genové Nicoloso di Castiello unos paños lombandos (ambos en ARV, Protocolos Desconocido, 11.178, del 27 y 30 de noviembre de 1296 respectivamente). Los dos aparecen como instnumensum debiti ex causa cambii, el eatadio enterior a la letra de cambio, en el que aún se recurre al notario, según R. de ROOVER, L'évolution de la lettre de change, XNVZ-XVIIIL siecles, Paris, 1953, especialmente Pp. $23-42$.
} 
armada en 1285 los recaudaba el banquero Guillem Arnau ${ }^{26}$. Igualmente la ayuda para el armamento de tres naves con las que socorrer al rey de Castilla, que se reclamaba a la universitat de Valencia en 1292, fue proporcionada inicialmente por Bernat Ferrer, Jaume de Sentboi y Bernat Planell ${ }^{27}$. Más tarde, los $30.000 \mathrm{~s}$. que se deblan conseguir en el reino para el desplazamiento de los emisarios reales a la corte papal, en 1295, se depositaron en la taula de Bernat Ferrer, mientras el monedatge del año 1302 lo recaudaba Bernat Planell23. Por otra parte, los cambistas participaban a menudo en las subastas de las bailfas locales, como Bernat de Llèmena, que en 1302 arrendaba todas las rentas de la de Dénia por 6.000 s. ${ }^{20}$; y pronto se introdujeron también en la financiación de los municipios valencianos, y sobre todo en la de la capital, donde se hallaban plenamente insertos en los resortes del poder local, llegando algunos a ocupar las más altas magistraturas ${ }^{30}$. Los cambistas constitulan de esta manera una especie de plutocracia plenamente inserta en la oligarquía local, al unir una fuerte presencia en la política ciudadana y los privilegios que sus relaciones con la Corona les proporcionaban.

Pero seguramente, tal y como ha observado S.P. Bensch en Barcelona, las fuertes sumas prestadas a los reyes, y las dificultades politicas del cambio de siglo, estarian en la base de los primeros temblores en la red bancaria valenciana, de la cual conocemos todavia solo una parte de sus efectos, y apenas nada de sus causas. Asi, es uno de los cambistas que

${ }^{20}$ ACA, Reial Cancelleria, reg. 56, fol. 49, 4 abril 1285.

${ }^{2 n}$ Loe dos primeroe facilition 20.000 s. cada uno y Planell 28.000 (ACA, Reial Cancelleria reg. 95, fols. 57 r. y v., 114 r. 137 v., y 179 r. y v.). En la mean de Ferrer, al margen del abisidio municipal, podian preatar cantidades los particulares, a los que es les prometín un incerts de $6 \mathrm{~d}$. por libre (2'5\%) (fol.137 v.).

${ }^{20}$ Reopectivemente ACA, Reial Cancelleria, reg. 101, fol. 53 v., 26 abril 195, y E. GONZALEZ HURTEBISE, op. cit. p. 203.

${ }^{29}$ ACA, Mestre Racional 2.694, fol. 1 v.

* La primera noticias de pretarmos al municipio pgr parte de carnbirtas datan ya de la decade de 1330 (J.V. GARCLA MARSILL- J. SAIZ, Ld transformaciones de la econombe mericipal de Valencia (ss. XIV-XV) (en prense). Pero con anteriorided ya encontramos numerosen nombres de banqueros en el consell en los primeros años de la centuria, por los menos once diferentes, que se repiten año tras año. Algunos incluso ortentaron el cargo de - juras, como Guillem Mir en 1308; Ramga de Poblet en 1310, 1313, 1322 y 1324; Bermat Phnell en 1311, 1319 y 1322; Pong de Soler en 1313, 1317 y 1323; Pere Guillem Catald en 1323; Mntew Bonencombes en 1325, etc. Mientre que Amau Guillem Catald fue justicia criminal en 1311; Guillem Mir lo fue en 1313 y 1326; Ramon Guillem Catald en 1316; y Ramon de Poblet justricia civil en 1329. 
hemos visto más ligado a los intereses de la monarquía, Bernat Ferrer, el primero del que nos consta que abandono el oficio y probablemente se di 6 a la fuga, ya que en 1299 sus principales clientes -cuatro mercaderes de Narbona y otro de Morlach- y su fiador, el también banquero Ponç de Soler, reclaman al rey que el Justicia de Valencia lo encuentre y le obligue a pagarles ${ }^{31}$. También Ferrer Oller, que hemos visto con anterioridad acusado de usura, habla cerrado su banco en 1299, puesto que aparece ya en la fuente como çaenrrere cambiador ${ }^{2}$. Pero quizás la bancarrota más importante, tanto por el volumen de capital malogrado como por la notoriedad del banquero y la espectacularidad del proceso, fue la de Ponç Fibla. En el mismo año fatidico de 1299 Fibla aparece ya ..propter fraudes per ipsum comissas in officio campsorie, captus in curie Valencie.., y diversos acreedores demandan que sean embargados sus bienes ${ }^{33}$. Un año más tarde los jurats de Valencia protestaban al rey porque dicho cambista habra eludido a la justicia municipal tonsurándose y alegando ser clérigo, ante lo cual protestaban porque según ellos Fibla ... sia lech et viva com a lech et en dbit laical, usan d'aytal offici com cambiador... No sabemos si finalmente esta argucia salvó al cambista arruinado, pero desde luego podemos afirmar que su quiebra arrastrarra a buena parte de la clase mercantil de la ciudad, ya que las autoridades locales cifraban en nada menos que 180.000 sueldos los haberes que éste tenfa en comandas y que se habian esfumado tras su abatiment".

Después le seguirfa en esta penosa lista de desastres bancarios Francesc de Pals. En su caso no fueron los préstamos directos a la monarquia los que causaron la bancarrota, sino su apoyo financiero a un noble, Jaspert, vizconde de Castellnou, el cual, primero vinculado a las empresas reales, y después por libre, realizo diversas campañas por el Estrecho y se asentó

\footnotetext{
${ }^{31} Y$ a en eatos momentos Bernat Ferrer aparece como olim campsor, lo que indica que ya no regents au coula; y wus clientes defraudedos eran Pere Fenoll, de Morlach; y Bemat Maylach, Bernat Sabater, Pong Amul y Guillem Cabrera, de Narbona (ACA, Reial Cancelleria reg. 114 , fol. 15 v. (19 octubre 1299).

sVid. supra note 18.

"Enere elloe el merceder de Perpignan Bemat d'Audiares, que reclarm 5.829 s., y loe ermadores de Barcelona Arnay y Guillem de Bartida 2.988 \&. y 11 d. berceloneses (ACA, Reial Cancelleria, reg. 144, fols. 125 r. y 143 v. reapectivamente; 17 y 23 de diciembre de 1299).

*ACA, Reial Cancelleria reg. 244 , fols. 228 v.- 229 r. (tranecrito por H. FINKE, Acta Aragonensia (Berlín-Leipzig 1908-1922, 3 vols.), doc. 533, tomo II, pp. 852-853). Citado tumbien por M. RrU, op. cit., p.204.
} 
durante un tiempo en Sevilla, como almirante mayor del rey castellano. Como más adelante veremos, los conflictos en que el vizconde se verfa envuelto, y los problemas económicos que éstos acarrearfan, provocarfan en poco tiempo el cierre de la taula de $\mathrm{Pals}{ }^{33}$. Como consecuencia de todos estos sucesos, en 1324 Jaime II se hizo eco de las quejas de los oficiales municipales, que afirmaban que ..paucis citra temporibus plures ex campsoribus civitatis predicte se sien abatuts cum magnis quantitatibus peccunie.., y volvió a insistir en la obligación de que los cambistas aseguraran sus bancos por valor de mil marcos de plata, y que presentaran fiadores ante el Justicia. No obstante, la orden establecia que los que prestaran dicha cantidad podrian poner una tapete o trapezet sobre su mesa, y quien no lo hiciera mostrar ra la madera desnuda; señal de que habla cambistas de condición más humilde, que se dedicarlan sólo al cambio manual, y serfan incapaces de conseguir esa cantidad. El símbolo externo del tapete servirfa asi como salvaguarda para los confiados clientes, que tendrfan una minima referencia sobre la verdadera solvencia de los banqueros ${ }^{36}$.

\section{EL NEGOCIO Y EL RIESGO: Auge y Caída de Francesc de Pals}

No se puede decir, sin embargo, que Francesc de Pals fuera uno de estos modestos cambiadores. Más bien este personaje se encuentra a mitad de camino entre ellos y los grandes banqueros de la época, puesto que

\footnotetext{
${ }^{33}$ El el mismo proceso 2 achace la quiebre a las "..magnas at arduas amissiones quas, de duobus annis citra, fecistis tam in terre quam in mari, et in partibus Castelle, et specialiter propter perigerius quod fuit factum vobis in partibus Castelle, ex mercibus a rendentibus in magna peccunie quantitaate ratione facti nobilis Jasperti de Castronovo.." (ARV, Justicia de Valencia 22, fol. 11 r.).

${ }^{36}$ Orden rocogida en el Aureum Opus Privilegionum Civilatis et Regni Valentie (cit.), fol. LXXIV v., p. 208 de la edición facsímil de M.D. CABANES, 5 kalendas aprilis 1324. Ya en 1283 Pedro el Grande había establecido la fianze de 1.000 marcos para los cambistas, pero no « debía de respehr eata noma cuando debió eer reformulada (lbidem, fols. XXX v. y XXXI r., p. 120). En cuanto a juramentos ande el justrcia, álo hemos podido encontrar uno en la documentación, y es el del hijo de Bernat Planell, Francesc, que en 1309 rustituía a au padre a frente del negocio, actuando entre otros como fiedor Bartomeu de Llemena, hermano del cambitt Bernat (ARV, Justscia de Valéncia 10 bis, fol. 13 r.). Las diferencias entre los auténticos banqueros y los simples cambista manuales es en realidad muestra del alto grado de espocialización propio de los centros financieros de importancia, y así aparoce claramente formulada en ciudadea italianas como Florencia o Venecia (vid. respectivamente Ch.M. de la RONCIERE, op. cit., p. 69; y R.C. MUEILER, op. cis., p. 160).
} 
tampoco lo encontramos nunca financiando a la monarquia, ni está entre los grandes prestamistas de la ciudad, ni llega a ocupar ningín cargo en el gobierno municipal; e incluso, los aproximadamente 80.000 sueldos que se le reclamaban no tienen comparación con las cantidades defraudadas por algunos de sus colegas a los que antes hemos hecho referencia. Por ello si sus negocios, incluso en visperas del hundimiento de su banco, sorprenden por su envergadura, su diversidad y su carácter internacional, cabe preguntarse cuál serfa el alcance de las empresas de esos grandes financieros de los cuales, por desgracia, poseemos menos referencias documentales.

En realidad la figura de Francesc de Pals nos serfa prácticamente desconocida de no conservarse las actas de su quiebra, ya que las referencias a él en otros documentos son casi inexistentes ${ }^{37}$. Por eso el estudio de sus negocios consistirá en una especie de "visión en negativo" a partir de los datos que nos proporciona el mismo proceso que contra el incoaron sus acreedores. A través de éste sabemos que Francesc de Pals estaba casado con Sibilia, hija de Joan Gil de l'Estela y de Tomasa, vecinos de la parroquia de Sans Pere Martir, los cuales en 1311 llegaron a un acuerdo con su yerno por el que le cedían la propiedad de su casa a cambio de que este los mantuviera durante su vejez. El trato consistra en que, por los 4.500 sueldos en que estaba valorada la vivienda, Pals les entregaba 494 sueldos al contado, se comprometra a proporcionarle otra casa libre de toda carga in civitate Valencie vel eius suburbiis, y les pagaría durante toda su vida una renta de 12 dineros diarios a Joan y 7 a su esposa. El impago de su violari a Tomasa, ya viuda en 1316, la convertiría en uno de los demandantes de su yerno, pidiendo que se conmutara esta pension por una cantidad de 2.000 sueldos al contado ${ }^{38}$. El cambista no debra habitar, de todas formas, en la vivienda que habla adquirido de sus suegros, ya que posela un suntuoso alberch en

\footnotetext{
"Únicamente lo hernos detectado en la correopondencia real en el aĩo 1315, cuando uno de ws deudores, Guillem de Milla, rocibe, por gracia del monarea, un aplazsmiento de dos años pare pagarte cien wueldos (ACA, Reial Cancelleria, fol. 134 v., Barcelona 7 kalendas augusti 1315).

*ARV, Jusifcia de Valincia 22, (en adelance citaremoe 2610 JV 22) fols. 58 r. -60 v. El ecuendo ee realizb el 14 kalendar januarii de 1311 (19 diciembre de 1310) ante el notario Pere Ferrer.
} 
la parroquia de Sant Nicolau, por el que sus acreedores conseguirfan nada menos que 15.000 sueldos $^{39}$.

Con todo, esa suma no fue suficiente para saldar sus deudas, puesto que ya de entrada se le demandaban $\mathbf{2 0 . 0 0 0}$ sueldos, a los que se añadieron otras cantidades cuando la quiebra del cambista se hizo pública mediante un pregón, y el justicia dio un mes de plazo para que se presentaran todos sus posibles acreedores ${ }^{0}$. En total se formalizaron al menos cuarenta y tres nuevas requisiciones, por un valor de 59.762 sueldos y 2 dineros, que se vinieron a unir a los 20.000 sueldos que inicialmente reclamaban las cuarenta y siete personas que hablan iniciado el proceso. La variedad en la cuantia de las deudas contrafdas es enorme. Desde los diez sueldos romanents de major quantitat que exigla el procurador del cavaller En Bellestar, a los 9.037 que pretendran cobrar los carniceros Arnau d'Esperandeu padre e hijo, se registra un amplio espectro de reclamaciones protagonizadas por miembros de la casi totalidad de los estamentos de la sociedad ciudadana ${ }^{41}$. Asi en la nómina de acreedores encontramos al clero, representado por las monjas de Santa María Magdalena y el mismo cabildo catedralicio, o por simples sacerdotes como Ponç de Pins; a individuos de la pequeña nobleza, como Arnau Escrivà, señor de Albuixec, o la viuda de Jaume d'Entença, señor de Benimàmet, y a personajes cortesanos, como doña Marina, ama de crla del infante Felipe de Castilla; a importantes judios de la aljama valenciana como Jucef Sibili, etc.. Pero sobre todo destacan los representantes de los oficios urbanos, en especial los más privilegiados, pertenecientes a la ma major y a la mitjana de la ciudad. De tal manera, entre los pocos acreedores que declaran su oficio tenemos un jurisperito, un notario, cinco mercaderes, otros cinco drapers, dos cambistas -Guillem de

\footnotetext{
"Lo edquirió Galceran de Riusec (JV 22, fol. 98). Sant Nicolau no era procieamente el barrio preferido de los cambistas, que se concentraban sobre todo en el de Santo Caserina, cence de aus bancos. Así, entre los consellers por parroquias que aparecen en los manuals del AMV, el único cambirta que es elegido por Sans Nicolau es Arnau Guillem Cacald en divereos años deade 1311 a 1322, con el cual Pals tuvo importantea negocios, como luego veremos, favorecidos probablemente por la relación de vecindad. En Sanla Caserina vivian en cambio los fiedores de Pals, Bemat Planell y Jaume Magraner, afí como Pere y Ramon Guillem Catald; y diemirados por otroe barrios eataban Romeu de Soler; (Sanea Maria y despues Sani Lorens), Bernat Ferrer y Metou de Boneecombes (Sant Esteve); Franceec Solanes (Sant Marth); y Jaume Sentboy (Sars Andreu) (datos tomedos de AMV, Manuals de Consells A-1).

${ }^{\circ}$ Le cantided de 20.000 sueldos roclamada en principio en JV 22, fol. 63. La crida del justrcia ee realiza el 8 de febrero de 1317 (Ibidem fol. 65 r.).

"Ambas cantidades en JV 22, fole 133 y 54 respectivamente.
} 
Sarrià y Ramon de Poblet-, un baxador y un blanquer exportadores de cueros, un sastre y cuatro importantes carniceros. Entre ellos destacan algunos de los linajes más señalados de la burguesía de la época, como los Valldaura, Rabassa, o Cardona. No obstante, si algo llama la atención en ese amplio listado es la cantidad de comerciantes provenientes del sur de Francia que mantenfan negocios con Francesc de Pals. Hasta nueve mercaderes de Narbona y cinco más de Perpignąn habran vendido en alguna ocasión paños al cambista, o estaban relacionados con él a través de algún contrato de cambio. Incluso tres de ellos serán elegidos síndicos para cobrar las deudas y repartirlas entre los demás acreedores, todo lo cual no hace sino confirmar la estrecha vinculación económica existente entre Valencia y Occitania en esta epoca ${ }^{42}$.

Naturalmente, los negocios que Francesc de Pals mantenfa con su amplia y variopinta clientela eran de muy diversos tipos, y a veces son diff́ciles de desentrañar a través de la simple presencia de una reclamación de numerario. No obstante, aunque no siempre es posible separarlos de forma nítida, intentaremos distinguir las operaciones puramente "bancarias" de aquellas más bien relacionadas con el comercio de distintos articulos, en las que también invertía con frecuencia este cambista. Entre las primeras es significativa la diferencia que se establece a la hora de que los acreedores renuncien a una parte de las sumas que se les adeudan, entre las comandes pures, cuyos titulares sólo pierden $2 \mathrm{~s}$. por libra, y altres dites e deutes de qualsevulla raho, de los que se dejaría de cobrar $6 \mathrm{~s}$. y $8 \mathrm{~d}$. por libra, es decir, nada menos que un tercio del tota ${ }^{43}$. Probablemente ello se deba a la diferente proporción entre capital y beneficios que existen en ambos casos, dado que en las comandas el interés serfa más reducido, y las ganancias de los inversores menores. Pero incluso en las comandas se debe distinguir

\footnotetext{
${ }^{42}$ Los mercaderes de Nartona que aparecen son Miquel d'Aigües; Bemat Berenguer, factor de Joan Arginayre; Jaume Boquer; Bemat de Saeyra; Ramon Peyter; Joan Raynart; Guillem Rosell; Bermat de Seyta y Jaume Bamo; y los de Perpignan Ramon Enmengau; Pere Pelegrí; Jaume Ganoyl; Guillem Imhert y Vidal Ramon. Miquel d'Aigües, Jaume Boquer y Pere Pelegrí fueron nombrados síndicos de los acreedores, aunque a los seis meses dejaron sus cargos porque "..Pelegrí sia en les parts de Roselló, et lo dit en Jaume Boquer sia en les parts de Nartona, et lo dit en Michel d'Aygües se'n vulla anar en les parts de Narbona..", por lo que cedieron sus poderes a los otros dos síndicos, los cambistas Bemat Planell y Jaume Magraner (JV 22, fol. 37, jueves 9 kalendas junii). Se trata por tanto de mercaderes no asentados, que realizan frecuentes viajes entre su tierra de origen y Valencia, y sólo nos consta de uno que tenga un factor instalado aquí.

${ }^{43}$ Dicho acuerdo aparece con frecuencia a lo largo de las actas de la quiebra, pero especialmente claro en el fol. $26 \mathrm{r}$.
} 
entre aquellas que funcionan como cuentas corrientes, en las que el depositario puede exigir en cualquier momento el reintegro del capital, pudiendo el cambista demorarse sólo durante quince dras sin incurrir en delito ${ }^{4} ; y$ aquellas en que si se establece un plazo fijo de devolución, como los seis meses en los que Martl del Castellar habla de recuperar 80 libras, o los catorce que debran permanocer en la taula de Pals 450 libras de Pere Arnau d'Esperandeu"s.

Compromisos más prolongados debran establecerse en cambio cuando, por ejemplo, se utilizaba el banco para depositar y administrar los bienes de una tutela o de una marmesorioto. En cualquier caso, los titulares de las cuentas solían realizar pagos a través de ellas. Mediante una de estas dites pretendía por ejemplo Arnau Escrivà pagar al obispo y al cabildo de Valencia su parte correspondiente del delme de su señorío de Albuixec en $1315^{47}$. Joan Montaner, por su parte, compró una casa a Romeu Dalmau, cuyo precio - 10.000 sueldos-- debra ir haciéndose efectivo por medio de dites en la taula de Francesc de Pals ${ }^{48}$. La insuficiencia de numerario de los cambistas hacia que a veces estas operaciones se complicaran bastante, recurriendo a las taules de otros banqueros. Quizás el caso más complejo de cuantos hemos observado sea la dita de 7.000 sueldos que hizo Mateu Costanç en favor de Eximén de Tovià en la mesa de Francesc de Pals, la cual se habla de hacer efectiva en el plazo de un año. En ese tiempo dicha cantidad fue transferida de la taula de Pals a la de Pere Viader, de ésta a la de Guillem Mir, y de esta última a la de Mateu de Bonescombes, que es quien aparece finalmente reclamándola ${ }^{49}$. En general, el carácter limitado de estos bancos medievales obligaría a una cierta interdependencia entre

\footnotetext{
"Es el caso del depósito de 150 l. que hace Guillamona, viuda de Pere Bonanat el 12 de marzo de 1315, en el que Francese de Pals ee compromele a devolver "..ad quindecim dies continue numerandos a prime amonestacione postquam a vobis vel vestris requisitus fuero.." (JV 2, fol. 49 r.).

${ }^{45} \mathrm{El}$ primero hizo el ingreso el 14 de julio de 1316, y era a pagar de wy en VI mesos (JV 22, fol. 70 v.); y la eegunda operación se realizó el XVII kalendes junii de 1315, y había de devolverse en los idus augusti de 1316 (Ibidem, fol. 55 v.).

"Ambos casos son constatables en el proceso: por ejemplo la tutela que ejercía Berenguer de Ralera sobre Franceaca, hija de Guillem Vivó de Boxeda, en la que cobrabe las herencias que la niña recibia (JV 22, fol. 85 r.); o los cobros que realizaban los albaceas del testamento de Bernat d'en Clapers (lbidem, fol. 102 r.).

${ }^{47} \mathrm{~V} 22$, fol. 87.

"Ibidem, fol. 78.

"JV 22, fol. 129 r. y v.
} 
ellos, necesaria para atender eventuales demandas de capital que sobrepasaran los haberes de que se disponia en caja. Asl, la transferencia que hemos observado anteriormente nos demuestra que el mismo Francesc de Pals tenia cuentas abiertas en las mesas de otros cambistas, y en efecto, en diciembre de 1316 algunos acreedores cobran lo que se les adeudaba ..dels diners que són en la taula d'en Mateu de Bonescombes, e que són del dit en Francesch de Pals.. ${ }^{\text {so }}$. Esta permanente relación entre los bancos tenfa también su lado negativo, y era que las dificultades por las que atravesara uno de los cambistas implicaban de forma inmediata a la práctica totalidad de la estructura financiera de la ciudad, como si de un efecto de vasos comunicantes se tratara ${ }^{\text {s1 }}$.

Pero la conmoción habria de notarse incluso fuera de las fronteras del reino, debido sobre todo a las operaciones de cambio con mercaderes de Narbona o Perpignan que quedaron malogradas por falta de capital del cambista. Por ejemplo las mercanclas que Simo de Joan, mercader valenciano, adquirio en Narbona de Joan Pelat valían 3.169 sueldos, los cuales debra hacer efectivos en Valencia Francesc de Pals al factor del narbones, Joan Mateu, pero el cambista sólo pudo reunir 407 sueldos $^{32}$.

Sin embargo, Pals no era un simple vértice pasivo en las operaciones financieras de los comerciantes languedocianos, sino que entre sus negocios no estrictamente bancarios destacaba el comercio con telas de importación de los centros manufactureros de Occitania. El volumen de las mercanclas compradas indica claramente que Pals adquirfa esos draps con la intención de revenderlos, y extraer con ello un beneficio en un comercio de artículos más bien de lujo. En efecto, de Jaume Boquó habla obtenido 42 draps de Narbona a 12 libras y 10 sueldos la pieza, lo que ascendra a un total de 9.242 sueldos; de Ramon Peyter otros 39 por 8.950 sueldos y 5 dineros; y

\footnotetext{
${ }^{50}$ Bbidem fol 48 v., 22 de diciembre de 1316.

s1 Igual aucode en Barcelona, donde lhs pérdidas de Berenguer de Fineatre arraenan también - otroo cambiadores, corno Barromeu Sendra y Pere de Sant Pere (S.P. BENSCH, op. cis., p. 324); 0 incluso en Florencia, donde las relaciones entre cambistas suelen centrase dentro del berrio donde eate ubicade la mean de cade uno (Ch.M. de la RONCIERE, op. cir., pp. 28-29).

s JV22, fol. 69 r. Al menos ocho operaciones aimilare ma se registran en eata fuente, por un valor total de 15.051 mueldos 7 dineros. Por el contrario hasth 233 contratos de cambio ha localizado G. ROMESTAN entre Valencia y las plaza occitanas cuyo pago ee debía realizar en exter úttima, entre 1316 y 1345. De ellos 61 ee harian en Montpellier, 60 en Nartom, 53 en Perpignan, y el reato en olsas ciudades menores (Les relasions commerciales entre Monupellier et Valence dans la première moitie du XIVZ siécle, VIII Congreso de Historia de la Corona de Aragón (1967), Valencia 1973, tomo II, Pp. 243-253.
} 
de Bernat de Seyta, 30 paños por 6.900 sueldos, a 11 libras y 10 sueldos cada uno. En todos los casos Francesc de Pals entregaba una cantidad a cuenta -aproximadamente el $10 \%$ en el caso de Bernat de Seyta- y se comprometia ante notario a pagar el resto al vencer un plazo establecido ${ }^{53}$. El mismo sistema utilizaba para la adquisición de cueros y cordobanes, pero en este caso el sentido del intercambio era inverso, ya que compraba la mercancla a productores o mercaderes locales, para luego exportarla ${ }^{\text {s4 }}$. E igualmente participaba en el comercio de algunas plantas aromáticas que crecian en el Pals Valenciano y encontraban mucha aceptación entre los paises del norte como condimento, tales como la batafalua -anis- y la alcarahuya -alcaravea en castellano-. El cambista compraba la cosecha a los mismos labradores de la región, como Bernat Octovia, de Sueca, que reclamaba 900 sueldos de un total de 1.000 que $\mathrm{Pals}$ le prometía pagar por algunas cargas de estos productos que le habla proporcionado ${ }^{35}$.

Los contactos de los cambistas con el mundo agricola no se limitaban sin embargo al comercio con los productos del campo, sino que solian ir más allá, dirigiendo a menudo parte de sus capitales a la compra de tierras o de instalaciones para la transformación de alimentos. El interés por el mercado inmobiliario no radicaba solamente en la seguridad de estas propiedades, verdaderos bienes-refugio de las fortunas mercantiles ante la incerteza de los negocios mercantiles; ni tan siquiera en la búsqueda de prestigio, sino que se puede afirmar que existra en él un verdadero ánimo de lucro. De ahl la predilección de los cambistas por la compra de molinos, cuya explotación

\footnotetext{
"2 Lo casos citados ee encuentran reunidos entre los folios 90 y 98 de JV 22. Sólo en el contrato de Bernat de Seyta se espocifica la cantidad que el cambirta habia entregado con

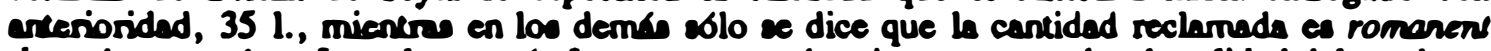
de major quantilas. Los plazos ma frecuentes son de seis meses, y cobre la calidad del producto collo ex habla de draps aineats o draps blencs de Narbons, excepto en un caso, en que se appecifica que el paño en de Xals d'Aloanyina, probablemento una imitación de tejidos de Chalone-wr-Mame, exin M. GUAL CAMURENA, Vocabulario del comercio medieval, Tarmagne 1968, PP. 457458.

SH El merceder de Valencia Pere Riera roclamabe por ejemplo 55 libras que Pals le debfa por anyram de conills (JV 22, fol. 100); mientras que Guillem de Corbere afimabe que ee le debian win 100 libres de 296 libres y 16 weldos que costabe el cordoud blanch que había vendido al cambirta y Androu Perongon, draper (Ibidem, fol. 99). Le imporancia del cuero valenciano en Perpignan y Montpellier en K.L. REYERSON, op. cit., p. 123.
}

ss JV 22, fol. 89 r. 
era una de las actividades más rentables del ciclo productivo agricola ${ }^{\star}$. En concreto Francesc de Pals tenfa a censo dos casals de molins en la vecina Russafa, sobre la acequia de Na Rovella, llamados de Sent Melio. Uno de ellos, lo pus jusd, era harinero y disponfa de dues rodes ab III caquaus; era por tanto una instalación importante y bien situada, ideal para abastecer la creciente demanda urbana. El otro, más pequeño -de una sola roda-, podía en cambio ser hasta más rentable, ya que estaba dedicado al arroz y la alheña, dos productos especulativos destinados al mercado exterior ${ }^{37}$.

Las principales empresas de este cambista estaban, desde luego, relacionados con ese mercado internacional, y especialmente con el vecino reino de Castilla. En efecto, Francesc de Pals, junto con otros dos banqueros valencianos, los hermanos Arnau y Ramon Guillem Catala, y dos mercaderes, Antoni Costans y Miquel Estrany, hablan arrendado en 1315 al maestre de Calatrava un peaje de dos dineros por carga de mercurio -argent viu-de Almadén, que se exportara hacia el reino de Valenciass. En sus negocios castellanos acabarfa sin embargo Francesc de Pals encontrando su ruina. Como ya hemos apuntado con anterioridad, este cambista intento extraer beneficios de las campañas navales en el Estrecho, y de la pujanza de Sevilla, apoyando económicamente las empresas de Jaspert, vizconde de Castellnou. Éste, que habla sido anteriormente procurador general del reino de Valencia, de 1300 a 1303, estuvo después al frente de la armada catalana que tomo Ceuta en 1309, y fue nombrado almirante mayor de Castilla por

\footnotetext{
Wor ejemplo, Bernat Planell compró la alqueria de Benimaclet y el baile le concedio licencia para contruir alle un molino (Anchivo de la Catedral de Valencia, Pergaminos no 352 (763 en el catrlogo de Olmos Canalda), 1 de junio de 1291). Siete años más tande eate miomo cambiata tenís también el dominio directo de otro molino fariner en el término de Ramble (ARV, Protocolos Desconocido, 11.179, 27 de mayo de 1298).

"St Sebemos de la exirtencia de eatos molinos por los pleitos que su dominio ocasionó entre los acroedores y antiguos socios de Francesc de Pals (JV 22, fols. 172-191). Delante de ellos, y dentro de la minma explotación habia también un terreno de nueve fanecades con un porche (fol. 191). La propiedad eminente de los molinos pertenecia al retor de Xive, Bernat de Alcoleja. Sobre la importancie del arroz y la alheña vid. respoctivamente nueatros cetudios La jerarquia de la mesa. Los sistemas alimenearios en la Valencia bajomedieval (Valencia, 1993); y Praduction et commerce des planses tinctoriales dans le Pays Valencien au Bas Moyen Age (comunicación al "2tme Congrta International. Partel, Indigo a autres plantes tinctoriales: peact, preserie at avenir", Toulouse 8-11 junio 1995 (en prenea)).

* Referencias a dicho artendaniares aparocen en ACA, Reial Cancelleria 156, fol. 66 r. (Barcelona, 19 junio 1315). En el proceso aparoce en el fol. 139 y as., y es uno de los pocos negocion que incluso desputa de ha quiebra genera beneficios y sirve pare cancelar algunas doude. El mercurio se usaba en la Eded Modia cobre todo pare el tratamiento de los motales prociocoo, con lo que los argensen de la ciudad y la minma ceca ecrian los posibles compradores (vid. M. GUAL CAMARENA, op. cif., p. 214).
} 
Fernando IV, conquistando Gibraltar ese mismo año ${ }^{39}$. Pals probablemente aprovechó la ocasion para establecer un factor en Sevilla, Domingo Buades, que además de proporcionar crédito a este noble, se constituyó en una especie de "cabeza de puente" de los mercaderes valencianos en la capital del Guadalquivir, de los que recibla mercancias para colocarlas en el mercado andaluz ${ }^{\circ 0}$. La dependencia con respecto a la hacienda de Castellnou debio seguir siendo, no obstante, muy importante, en un caso similar, aunque a una escala mucho más reducida, a la vinculación entre las grandes compañias italianas y los reyes de Inglaterra ${ }^{61}$. Y como les ocurriera a estos gigantes financieros, la suerte del banquero comenzo a cambiar cuando la del noble al que se habla ligado economicamente dio un inesperado giro negativo. En mayo de 1315 Jaspert de Castellnou es acusado por el concejo de Sevilla de haber atacado al abad de Santa Cruz, por lo que, invocando el derecho de quema, son embargados los bienes de algunos mercaderes de la Corona de Aragon ${ }^{62}$. Perdido el favor de ambos monarcas, la situación del vizconde debió ser bastante crítica, más cuando siguió manteniendo una ruinosa guerra feudal contra Pere de Fenollet; y sus acreedores, incluido Francesc de Pals, se debieron encontrar ante la imposibilidad de recuperar las sumas que le hablan proporcionado, lo que acabaria por desencadenar una sonora bancarrota ${ }^{63}$.

$$
* * *
$$

50. ZURITA, Anales de la Corona de Aragón, libro 5, tomo 2, Zaragoza, edición de 1979 a cargo de A. CANEUUS, caps. LXXIX y LXXXIIII, pp. 715 y 725.

${ }^{\infty}$ Asi por ejemplo, Buades recibió de Pere Despont hacia 1315 I costal de pebre ensarpallat e partida de safrá e montonacs, cuyo precio, 2.381 morabatines menos 1 dinero, envió a Francesc de Pals, y Despont lo reclamaba el 19 de junio de 1317 (JV 22, fols. 2-4).

${ }^{61}$ Vid. a eate respecto, además de la bibbliografía ya citada, C. CIPOLLA, El gobiemo de la moneda. Enscyos de historia monetaria, Barcelona, 1994 (original en Bolonia, 1990), especialmente las pp. 147-169; y E.S. HUNT, The medieval Super-companies. A studty of the Penues company of Florence, Nueva York, 1994.

-ACA, Reial Cancelleria 156, fol. $10 \mathrm{v}$. . Barcelona 20 de mayo de 1315. La carta va dirigida a los hombres buenos de Sevilla y en ella el rey protesta porque Jaspert de Castellnou no es súbdito suyo, sino del rey de Mallorea, y no puede actuar contra él, al no poseer tierras en su reino.

${ }^{63}$ En el fol. $11 \mathrm{r}$. del proceso, antes citado (vid, supra nota 35). Se explica que comenzaron las difícultades es dos años antes de la redacción del proceso (1314-1315), lo que coincide con el incidente de Jaspert de Castellnou antes señalado. La guerra contra Fenollet en Gran Enciclopedia Catalana, Barcelona 1986, tomo 6, p. 461. 
Las actas de la quiebra reflejan en realidad el intento, sobre todo por parte de los fiadores del cambista arruinado, de poner un poco de orden entre los demandantes para ir estableciendo la forma y el turno en que estos deberfan cobrar lo que se les debra. El volumen que se compilo da muestra de la extensa red de intereses económicos que se urdía en torno a la mesa de estos incipientes banqueros en un centro como Valencia, que comenzaba a ocupar un lugar importante en el escenario financiero del Mediterráneo occidental medieval.

Cuando apenas habla transcurrido medio siglo desde la conquista cristiana, en Valencia se habra gestado ya una clase bancaria de cierta entidad, que participaba activamente en el comercio internacional, contribura a la liquidez de la Corona y jugaba un papel de primer orden en el desarrollo economico e institucional del reino. Las técnicas empleadas, la complejidad de sus compañlas, que contaban incluso con factores en plazas extranjeras, y el poder que llegaron a adquirir en el espacio politico local, demuestra la importancia de estos cambistas locales. Por supuesto, a un nivel muy inferior al de las grandes compañfas italianas del momento, los banqueros valencianos iniciaron ya de forma autoctona su andadura profesional desde el mismo momento de la incorporación al Occidente cristiano, y contribuyeron a la creación de un espacio economico mediterráneo, actuando como verdaderos mediadores de capital entre zonas como el pais de Oc, Italia y Castilla.

Sin embargo la diffcil coyuntura en torno a 1300 pareció quebrar el crecimiento sostenido del mundo financiero valenciano, y dejo por primera vez al descubierto sus deficiencias. Las circunstancias políticas debieron ser determinantes para este primer reves, tanto a nivel internacional, como, sobre todo, en el ámbito concreto de la Corona de Aragon. En efecto, como ya argumentara S.P. Bensch para Barcelona, la expansion militar y mercantil por el Mediterráneo atraveś por momentos delicados tras la pérdida de Sicilia en el tratado de Agnani de $1295^{\circ}$, y ello fue la causa de las penurias económicas de la monarquia, que afectaron de forma negativa a los banqueros que la sostenfan. La política agresiva que en estos años protagoniza el poder real, como forma de catalizar a la sociedad hacia la formación de un nuevo Estado más autoritario, se plasma en numerosas campañas, como la de Almerfa, que algunos miembros de la nobleza intentan aprovechar como una vía para aumentar su riqueza y prestigio. El fracaso de

\footnotetext{
"W.P. BENSCH, op. cit. pp. 324-327.
} 
aventuras personales, como la de Jaspert de Castellnou, arrastrarfa también de esta manera a los cambistas que habran arriesgado sus capitales en ellas en espera de cuantiosos beneficios.

Sin duda los financieros valencianos, y en concreto Francesc de Pals, sobreestimaron su capacidad económica, espoleados por la tendencia expansiva que vivía la economía valenciana en estos momentos, e invirtieron de forma desproporcionada en negocios demasiado dependientes de la coyuntura polftica, y que estaban muy por encima de sus limitadas posibilidades de captación de capital. La crisis no fue sin embargo definitiva, sino más bien

un traumático reajuste en una evolución que seguirfa siendo expansiva a largo plazo. Las quiebras de principios del siglo XIV dieron paso a una especie de "relevo generacional" entre los cambistas valencianos, uniéndose a algunos linajes que sobrevivieron, como los Solanes, Soler, Guillem Català, etc., nombres nuevos como los Valleriola, Donat o Cedrelles, que se caracterizaron sobre todo por mantener vínculos más estrechos con las grandes compañfas extranjeras, en una época en que comenzaba a vertebrarse el mercado europeo.

\section{RÉSUMÉ}

La banqueroute du changeur valencien Francesc de Pals, l'anne 1316, est une plus d'une série de brisures bancaires produites par tout la Méditerrance occidentale, at spcicialement a Valence, depuis les dernières années du XIIle siecle. La conservation du procès par lequel ses créanciers s'ont distribuit son patrimoine sert a analyser le naissant monde financier de la cité de Valence a peine demi sizcle aprés son intégration au sein de l'Europe chretienne. De cette façon, les changeurs autochtones avaient développé, dejà en cette époque, une réseau d'affaires que comprenait des fluides relations avec l'Italie et le pays d'Oc, at ils avaient méme établi des facteurs en Seville. Précisément, la liaison de Francesc de Pals avec les entreprises militaires et piratiques que le noble Jaspert de Castellnou a réalise dans l'Étroit de Gibraltar est le commencement de sa ruine.

\section{SUMMARY}

The bankruptcy of the valencian moneychanger Francesc de Pals, in 1316, was snother one in the long sequence of banking failures that happened in the Western Mediternmean Sea, and specially in Valencia, by the end of XIIlth Century. The conservation of the process where his creditors shared out his inheritance is the staring point for a 
breakdown on the rascent financial world of Valencia only half a Century after its integration to Christian Europe. So, native moneychangera had already developped at this time a businesa network that included important relations with Italy and the South of France, and they had even setuled agents in Seville. The connexion betwoen Francesc de Pals and the militar and piratical enterprises that the noble Jaspert de Castellnou carried out in the Straits of Gibraltar was just the start to his final collapse. 